\title{
Self-Similar Hot Accretion Flow onto a Rotating Neutron Star: Structure and Stability
}

\author{
Mikhail V. Medvedev ${ }^{1}$ and Ramesh Narayan ${ }^{2}$ \\ ${ }^{1}$ CITA, University of Toronto, Toronto, Ontario, M5S 3H8, Canada \\ ${ }^{2}$ Harvard-Smithsonian Center for Astrophysics, 60 Garden Street, Cambridge, MA 02138
}

\begin{abstract}
We present analytical and numerical solutions which describe a hot, viscous, two-temperature accretion flow onto a rotating neutron star or any other rotating compact star with a surface. We assume Coulomb coupling between the protons and electrons, and free-free cooling from the electrons. Outside a thin boundary layer, where the accretion flow meets the star, we show that there is an extended settling region which is well-described by two self-similar solutions: (i) a two-temperature solution which is valid in an inner zone $r \leq 10^{2.5}$ ( $r$ is in Schwarzchild units), and (ii) a one-temperature solution at larger radii. In both zones, $\rho \propto r^{-2}, \Omega \propto r^{-3 / 2}, v \propto$ $r^{0}, T_{p} \propto r^{-1}$; in the two-temperature zone, $T_{e} \propto r^{-1 / 2}$. The luminosity of the settling zone arises from the rotational energy of the star as the star is braked by viscosity. Hence the luminosity and the flow parameters (density, temperature, angular velocity) are independent of $\dot{M}$. The settling solution described here is not advection-dominated, and is thus different from the self-similar ADAF found around black holes. When the spin of the star is small enough, however, the present solution transforms smoothly to a (settling) ADAF.

We carried out a stability analysis of the settling flow. The flow is convectively and viscously stable and is unlikely to have strong winds or outflows. Unlike another cooling-dominated system - the SLE disk, — the settling flow is thermally stable provided that thermal conduction is taken into account. This strong saturated-like thermoconduction does not change the structure of the flow.
\end{abstract}

\section{THE SETTLING FLOW}

At small mass accretion rates, $\lesssim 10^{-2}$ of the Eddington rate, black holes (BHs) accrete via an ADAF - a hot, two-temperature, radiatively inefficient, geometrically thick, advection-dominated accretion flow [1,2]. In contrast, accretion onto compact stars, e.g., a neutron star (NS) may occur via either an ADAF of a settling solution [3]. The latter corresponds to strongly rotating stars only. In the settling flow the rotational energy is extracted from the star via viscous torques in the boundary layer where the flow meets the star surface. The extracted energy heats 
the flow and ultimately escapes from the flow as free-free radiation. In addition, viscosity extracts angular momentum from the star as well. In the stationary flow, this angular momentum must be transported though the flow to the outermost radii, where it goes into the ambient medium. It is this huge angular momentum flux $\dot{J}$ which modifies the entire structure of the accretion flow and makes it drastically different from an ADAF. Note that no angular momentum may be extracted from a BH horizon by viscosity. Thus a viscous settling flow may exist in NS systems and not in BH systems.

The structure of the steady, rotating, axisymmetric, quasi-spherical, twotemperature settling flow has been found analytically and confirmed numerically [3]. We use the height-integrated form of the viscous hydrodynamic equations with the Shakura-Sunyaev-type viscosity parametrized by dimensionless $\alpha$. We assume viscous heating of protons, Bremsstrahlung cooling of electrons and Coulomb energy transfer from the protons to the electrons; we neglect Comptonization but include thermal conduction in the form discussed in the next section. In the inner zone
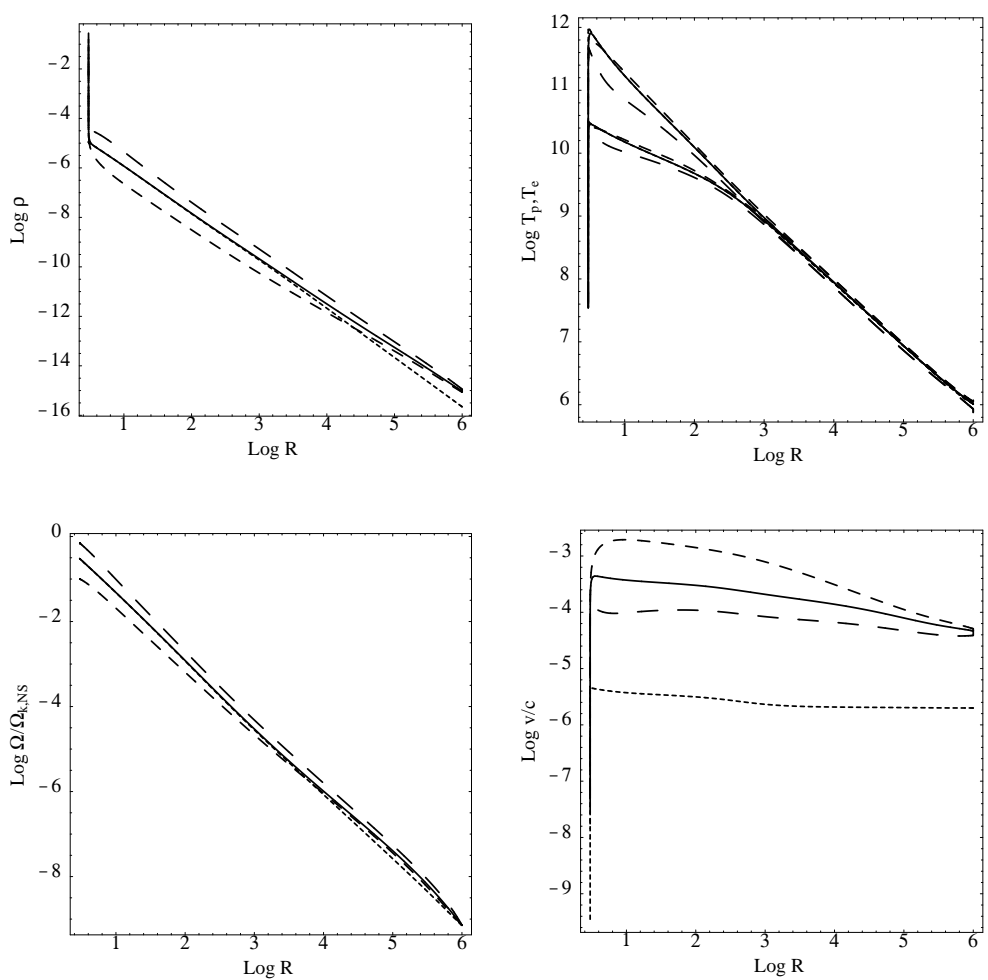

FIGURE 1. Profiles of density $\rho\left(\mathrm{g} \mathrm{cm}^{-3}\right)$, proton temperature $T_{p}\left({ }^{\circ} \mathrm{K}\right)$, electron temperature $T_{e}\left({ }^{\circ} \mathrm{K}\right.$ ), angular velocity $\Omega$ (in units of the Keplerian angular velocity at the NS radius $R_{N S}$ ), and radial velocity $v$ (in units of $c$ ) for accretion flows with $\alpha=0.1$ and $(\dot{m}, s)=(0.01,0.3)-$ solid line, $(0.0001,0.3)$ - short-dashed line, $(0.01,0.1)$ - medium-dashed line, $(0.01,0.7)$ - long-dashed line. 
$r<10^{2.5}$ ( $r$ is in Schwarzchild units, $R_{S}=2 G M / c^{2}$ ), the flow is two-temperature with the density, proton and electron temperatures, angular and radial velocities scalings as

$$
\rho=\rho_{0} r^{-2}, \quad T_{p}=T_{p 0} r^{-1}, \quad T_{e}=T_{e 0} r^{-1 / 2}, \quad \Omega=\Omega_{0} r^{-3 / 2}, \quad v=v_{0} r^{0},
$$

where $\rho_{0}, T_{p 0}, T_{e 0}, \Omega_{0}, v_{0}$ are functions of $M, \alpha$ and the star spin $s=\Omega_{*} / \Omega_{K}\left(R_{*}\right)$, and $\Omega_{K}(R)=\left(G M / R^{3}\right)^{1 / 2}$ is the Keplerian angular velocity. In the outer zone $r>$ $10^{2.5}$, we have $T_{e}=T_{p} \propto r^{-1}$ and the same other scalings. This self-similar solution is valid for the part of the flow below the radius $r_{s}$ related to the mass accretion rate $\dot{m}$ (in Eddington units, $\dot{M}_{\text {Edd }}=1.4 \times 10^{18} \mathrm{~m} \mathrm{~g} / \mathrm{s}$, and here $m=M / M_{\odot}$ ):

$$
\dot{m}<2.2 \times 10^{-3} \alpha_{0.1}^{2} s_{0.3}^{2} r_{s, 3}^{-1 / 2},
$$

where $r_{s, 3}=r_{s} / 10^{3}, \alpha_{0.1}=\alpha / 0.1$, etc.. The numerical solution of the hydrodynamic equations with appropriate inner and outer boundary conditions is represented in Figure 1. It is in excellent agreement with the self-similar solition (1).

There is a remarkable property of the settling flow: all quantities, e.g., $\rho, T_{p}$, etc., except $v$, are independent of the mass accretion rate $\dot{M}$. This happens because the gravitational energy of the accreting gas is much smaller than the energy extracted from the rotating star. It is this energy which dominates the flow luminosity. We should also remark that the settling flow is more similar to a steady, radiative cooling-dominated "atmosphere" rather than to a rapidly infalling flow: the radial infall velocity is constant and is much less then the free-fall velocity $v / v_{\mathrm{ff}} \propto r^{1 / 2} \rightarrow 0$ as $r \rightarrow 0$. The structure of the settling is very sensitive to the rotation rate of the central star. As the angular velocity decreases below few percents of the Keplerian value, the settling flow smoothly transforms into a conventional ADAF solution, as represented in Figure 2.

It was shown that the settling flow is (i) convectively stable and (ii) may not have strong winds and outflows (the Bernoulli number is negative) if the adiabatic index satisfies

$$
\gamma>\frac{3\left(1-s^{2} / 2\right)}{\left(2-s^{2} / 2\right)} \sim 1.5 .
$$

Other properties of the settling flow are discussed elsewhere [3,4].

\section{THERMAL STABILITY OF THE SETTLING FLOW}

The settling flow is cooling-dominated. Thus it is similar to the ShapiroLightman-Eardley solution [5] which is known to be thermally unstable [6]. Hence, our settling solution may be unstable as well. To study the thermal instability in sheared circular accretion flows we use the shearing sheet approximation with the velocity given by 


$$
\mathbf{V}_{0}(x)=2 A x \hat{y}
$$

where $2 A=d \mathbf{V}_{0} / d x$ is the shear frequency, $x, y, z$ are the radial, azimuthal, and vertical coordinates, and "hat" denotes a unit vector. We assume that there is a Coriolis acceleration due to $\Omega=\Omega \hat{z}$. The vorticity and epicyclic frequency are then $2 B=2 A+2 \Omega, \kappa_{\text {epi }}^{2}=4 \Omega B$. For a Keplerian-type flow, $\Omega \propto R^{-3 / 2}$, which is the case for both the settling and SLE solutions considered below, one has $2 A=-(3 / 2) \Omega$ and $2 B=\Omega / 2$. We assume that perturbations have only $s$ component, which corresponds to axisymmetric perturbations. We ignore motions in $z$ direction. We use hydrodynamic equations with thermoconductive flux.

The settling flow is hot (sub-virial), so that the mean-free-path (of both electrons and protons) is larger than the size of the system. Hence the conventional Spitzer theory fails. Without collisions but in the presence of magnetic fields electrons stream freely along the field lines, therefore the parallel heat flux remains large. In contrast, transverse heat flux is greatly reduced in a magnetic field because electrons are tied to the field lines on the scale of the Larmor orbit and cannot move across the field lines too far. In a tangled field, however, electrons can jump
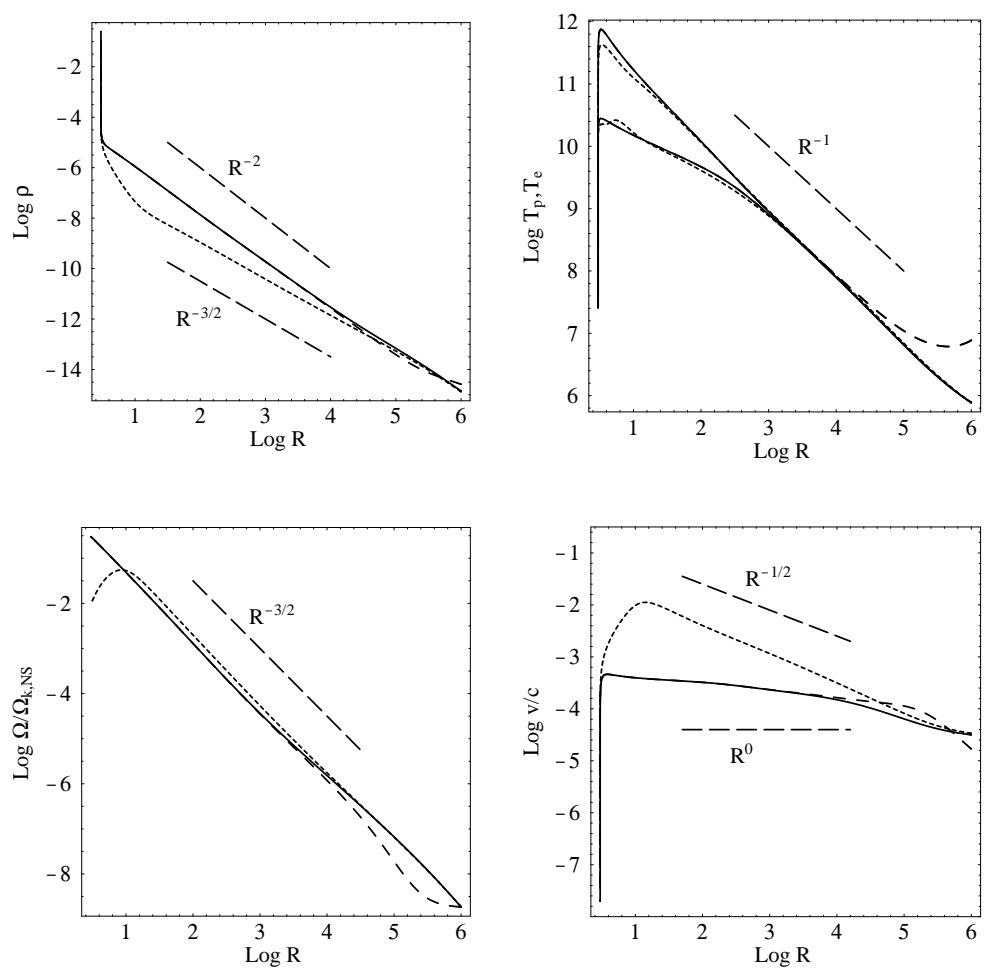

FIGURE 2. Same as in Fig. 1 for $\gamma=4 / 3$ and $s=0.3$ (solid curve) and $s=0.01$ (dotted curve). The self-similar slopes for an ADAF flow and a settling flow are shown for comparison. The long-dashed curves represent the same solution as the solid curve, but with ten times higher temperature at $R_{\text {out }}$. 
from one field line to another and thus transfer heat across the field [7]. The average thermal conductivity in tangled fields is

$$
\kappa_{B} \simeq n k_{B} v_{T} l_{B} \vartheta \simeq 10^{-2} n k_{B} v_{T} R \xi_{-1} \vartheta_{-1},
$$

where $l_{B}=\xi R$ is the correlation scale of the magnetic fields set by flow turbulence and $\vartheta<1$ takes in to account that only a fraction of all particles may pass though the magnetic barriers. The rest of them will remain trapped in magnetic wells and, hence, will not transport energy to large distances $\gg l_{B}$. The thermoconductive flux is

$$
q_{\mathrm{cond}}=-\alpha_{c} \frac{\rho c_{s}^{2}}{\Omega_{K}} \frac{d c_{s}^{2}}{d x}, \quad \text { where } \quad \alpha_{c} \simeq \frac{R}{H} \xi \vartheta F(e, p) \simeq 10^{-2} \xi_{-1} \vartheta_{-1} F(e, p)
$$

is the "alpha-prescription" thermo-conductivity coefficient and $F(e, p)[1 \leq$ $F(e, p) \lesssim 15]$ takes into account that conduction may be dominated by the protons or the electrons, depending on the flow conditions. Here we used that $v_{T} \simeq c_{s e}$ and $H / R \sim c_{s} / v_{\mathrm{ff}} \sim c_{s} / \Omega_{K} R$, where $H \sim R$ is the accretion disk scale height.

Now, from hydrodynamic equations for the perturbations, we obtain the dispersion relation

$\omega\left[\frac{\omega}{\gamma-1}+\frac{i(n-1)}{\tau_{\text {cool }}}+\frac{i k^{2} R^{2}}{\tau_{\text {cond }}}\right]\left(\omega^{2}-\kappa_{\text {epi }}^{2}-k^{2} c_{s}^{2}\right)-\omega\left[\omega+\frac{i(2 B / A-1)}{\tau_{\text {cool }}}\right] k^{2} c_{s}^{2}=0$,

where $\quad \tau_{\text {cool }}=\frac{4}{\left(9 \alpha s^{2}+2 \alpha_{c}\right)} \Omega_{K}^{-1} \simeq \frac{2}{\alpha_{c}} \Omega_{K}^{-1}, \quad \tau_{\text {cond }}=\frac{3}{\alpha_{c}} \Omega_{K}^{-1}$.

In the large- $k$ limit, (7) yields the growth rate of the thermal mode. The stability criterion $\operatorname{Im} \omega<0$ is $\left(\right.$ for $\alpha s^{2} \lesssim \alpha_{c}$ )

$$
k R>\left[\frac{\tau_{\text {cond }}}{\tau_{\text {cool }}}\left(2-n-2 \frac{B}{A}\right)\right]^{1 / 2}=\left[\frac{26 \alpha_{c}}{9\left(9 \alpha s^{2}+2 \alpha_{c}\right)}\right]^{1 / 2} \simeq \sqrt{\frac{13}{9}} \simeq 1.2,
$$

that is, thermal modes with $k R \geq 2$ are stable. Whether the mode $k R=1$ is stable or not cannot be reliably determined from our local approach. A global stability analysis is necessary to properly account for the effects of geometry and curvature on the eigenmode structure. Finally, we conclude that the settling flow is very likely stable.

\section{REFERENCES}

1. Narayan, R., and Yi, I., Astrophys. J. 428, L13 (1994)

2. Narayan, R., and Yi, I., Astrophys. J. 452, 710 (1995)

3. Medvedev, M. V., and Narayan, R., Astrophys. J. 554, 756 (2001)

4. Medvedev, M. V., in Proceedings of the 20th Texas Symposium, eds. J. C. Wheeler and H. Martel (2001)

5. Shapiro, S. L., Lightman, A. P., \& Eardley D. M., Astrophys. J. , 204, 187 (1976)

6. Piran, T., Astrophys. J. , 221, 652 (1978)

7. Rechester, A. B., \& Rosenbluth, M. N., Phys. Rev. Lett., 40, 38 (1978) 